which was situated on the posterior wall in the median line, showed no disposition to heal. In March, 1894, he became infected with syphilis, disposition to heal. In March, 1894, he became infected with syphilis, and was placed upon mercury. To my surprise the bladder symptoms gradually diminished, the penile pain rom which he had suffered so long disappeared, the urine cleared, and in October, 1894, I noted that he was week (April week (April, 1896). On the posterior wall of the bladder, low down, was a florid, gelatinous, keloid-like scar. Radiating from its puckered centre and diverging like a star were prominent thin-edged ridges. Obviously the scar tissue in contracting had drawn into folds the loose mucous The rest of the bladder was in perfect health, 12 ounces pacity

The best form of treatment in the first stage is sandal oil, followed by maltine and cod oil, small doses of mercury at night, liberal diet, and fresh air. Should the ulcer prove obstinate, curettage will have to be performed.

Treatment in the Second Stage.-I know of no reliable medicine when once the ulcer has become encrusted with lime phosphate (second stage). Vesical washes are better. Lactic acid from I per cent. or nitrate of silver or acids are of use, but they are very trying and uncertain. The best way is to thoroughly scrape the surface of the ulcer. In men this must be done perineally or suprapubically. In women, who seem to be particularly prone to this form of ulcer, curetting is easy enough. A 28 (Fr. gauge) speculum is introduced in the Trendelenburg position, and the ulcer scraped every other day under electric light. It is surprising how rapidly cases in the earlier stages improve after this treatment. I have known a fortnight a sufficient time to cure an ulcer of months' duration. But when the case is advanced much patience and perseverance is necessary. One lady, who was sent me by Dr. James Tuxford, of Boston, in October, 1891, was almost entering the third stage when first I saw her. Her bladder only retained one ounce of urine and that with difficulty. Her sufferings were extreme. She had a lime-encrusted ulcer in the usual position on the posterior wall, and a "contact" ulcer on the anterior wall (Fig. 4). I scraped these ulcers, with and without the electric light, fifteen times, and I am glad to say she was able after six months' treatment to hold water for three hours in the day and longer at night, and is now without pain or discomfort. When I last examined her with the cystoscope the ulcers had healed.

Fallacy.-It must be distinctly understood that the solitary simple ulcer repays active curettage, but tuberculous ulceration resents such a proceeding. I fear surgeons often do more harm than good by interfering with the tuberculous bladder. I suspect that the very favourable reports which have appeared from time to time in the press of cure of tuberculous ulcer of the bladder after curettage and drainage have, in some instances at least, been attacks upon the simple solitary ulcer which so nearly resembles the tuberculous in its symptoms, but which differs from it so markedly in its prognosis.

\section{THE CAUSE OF MALARIA.}

Extract from an Address delivered at the Grant Medical College, Bombay, April 4th, 1896.

By Surgeon-Lieutenant-Colonel E. LAWRIE, M.B., Residency Surgeon, Hyderabad.

[AFTER some preliminary remarks, the lecturer showed a case of recurring malarial fever, with enlarged spleen, in whose blood Laveran's bodies were found. Criticising the figures of these bodies, which have appeared in English medical journals and in other works, he characterised them as " fanciful pictures;" some of them being "drawn from the imagination, others," he said, "especially the rosette form, are caricatures of vacuoles and disintegrating blood cells." Others he considered as being "probably crenating red cells." "It is not surprising," he continued, "that the theories of malarious fever, founded upon the assumption that Laveran's bodies are animal parasites living in the blood are fantastic and fine spun." After a brief sketch of some of the more salient features of the modern doctrine of malarial disease, Dr. Lawrie proceeded, in the following words, to give his own views as to the nature of Laveran's bodies:- ]

Healthy blood contains two kinds of cells, the red and the white. The red cells convey gases to and fro between the lungs and the tissues. Their functions are urgent and al:- important, and admit of no interruption or relaxation. Hence, among other marvellous qualities, they possess an elasticity which is absolutely perfect; nothing stops their career, and they dash through the narrowest channels, round the sharpest corners, and past obstacles of any and every kind without the smallest check or hindrance. The mobility, energy, and outward activity of the red cell give it the stamp of what Drummond calls " maleness;" it is an organism principally concerned with nutrition. The white cells are very different from the red; when fully developed they appear to me to be filled with granules, which are minute living protoplasmic cells, and many of them contain large clear spaces, hitherto regarded as nuclei, which must, in my opinion, either be fertilising or nutrient areas; most likely they hold reserves of protoplasm. I throw it out as a hint to the profession that they supply the blood and all the tissues each with the particular kind of protoplasm and young protoplasmic cells suitable for its own special requirements. They incorporate in their own being the two main activities of all living things, nutrition and reproduction, and their linternal activity or passivity, and the fact that they teem with young cells and granules, indicates that they are the female, in the sense of being the maternal cells of the blood. The white cells are never in a hurry like the red; they loiter about in the capillary system, and stick to the walls of vessels and pass through them, shedding their contents either in the blood or in the tissues. The white cells have special movements of their own, which consist of varied activities of their protoplasm. These cause the cell itself to change its shape and position, and they produce intracellular movements of the granular contents, which are seen sometimes moving slowly, and at others actively "swarming" with the identical movement described by Laveran in connection with his malaria bodies as that of the "disturbance of boiling water."

The white cells of the blood further have the power of throwing out pseudopod processes which, if thread-like or filamentous, are called cilia or flagella, and these protoplasmic offishoots can be detached, and lead for a time an independent existence. The ordinary forces of the circulation, the heart and the elasticity of the vessels, are not sufficient to account for the whole of the movement of the blood in the capillaries, and it seems, therefore, highly probable that both the intracellular circulation called "swarming" and the evolution of "pseudopoda," which is a primitive force of the circulation, are what Pettigrew long ago described as those occult forces which regularly assist in the peculiar circulation of the blood cells and of the blood plasms in the capillary system.

A most important point, bearing on the origin of Laveran's bodies, is the source from which the blood cells are derived. They are largely manufactured or elaborated in the spleen or liver. - In man the red cell is not nucleated but in the human embryo it is nucleated, and it is also nucleated in the frog, and in the frog the nucleus is clearly the intracorpuscular stage of the white cell.

No. I is a photograph of a red and white cell of frog's blood, copied from Landois and Stirling's book, and it is evident that the white cell is identical with the nucleus of the red. I was led to make an examination of the blood of the frog by an accident. In accordance with letter No. 3.278 from the Government of India Foreign Department to the Resident at Hyderabad, covering a paper by Dr. Patrick Manson on the Nature and Significance of the Crescentic and Flagellated Organisms in Malarial Blood, the medical officers serving under the orders of the Resident at Hyderabad were directed to make observations on the "organisms of malaria, and were specially enjoined to pay particular attention to the life history of the parasite." At the time this letter was received in my office we had been working for several months at the microscropic appearances of the blood in malaria, and it appeared to me that before Laveran's bodies could be properly assumed to be parasites they must be shown to have an independent existence outside the human body, and that a likely habitat for them, if they had such an existence, would be the body or the blood of frngs, frogs being known to flourish where malaria abounds. The examination of the blood and of the liver and spleen of frogs was undertaken while the animals were alive (anæsthetised). In the blood and in the 
free protoplasm of the liver and spleen we discovered, to our astonishment, not Laveran's bcdies, but their perfect and complete analogues. In the liver and spleen of frogs were found small protoplasmic cells and granules, identical with those contained in the white blood cells in man, "swarming" with the same movement of boiling water which Laveran had discovered in his organisms, and which we had discovered in the leucocyte. We also found blood cells in every stage of development, and the white cell could be traced from its origin as an intracorpuscular body, when it sometimes becomes the nucleus of the red cell and at others becomes a free and independent leucocyte, until it changes into an extracorpuscular body by bursting through and incorporating with itself the red cell. The white cell in the frog has to serve the purpose of nutrition of a simple slow, cold-blooded amphibious animal, and the processes of its development are correspondingly simple, and easy to follow.

In man the blood cells are unquestionably formed on the same lines as those of the frog, but the nutritive processes are so complex, and the difficulties of examining the blood glands during life are so insuperable, that it is correspondingly difficult to follow the stages of the developmental processes. It is a curious fact, however, that wherever difficulty arises in tracing out the analogy between the two assistance is afforded by Laveran's bodies.

Lastly, the "swarming" movement of the protoplasm and of the young protoplasmic cells in the spleen of the fro indicate that there is a double circulation in that organ, and very probably in the liver also. This view receives confirmation from the discovery of Roy, extended by Schäfer and Moore, of the rhythmic contractility of the spleen. I mention this here because the double circulation in the spleen may turn out to have an interest bearing on the diurnal variations of the temperature in health, as well as on the periodicity of malarious fever when those variations are exaggerated.

In the light of the above physiological facts we are in a position to consider the actual value of the characteristic features of Laveran's bodies, which have been made the foundation for the belief that they are parasites living in the blood.

I. They are intracorpuscular in one stage of their existence. But the white cells of the frog's blood, which are the analogue of the human white blood cell, are at one period of their existence intracorpuscular bodies, and no one would dream of calling them parasites.

2. Laveran's bodies are at another stage of their life extracorpuscular. So are the white cells of the blood.

3. The free body changes its shape and becomes oval or crescent-shaped. The crescent is supposed to be a parasite and to "devour" the red blood cells. There is nothing remarkable about the change of shape of the crescent. All cells which contain granules tend to become spindle-shaped when the granules collect in the centre of the cell, and round again when they spread out to the margin, and the red blood cell of the frog in which the granules of the nucleus are collected in a compact mass in the centre is itself spindleshaped. The resemblance between the red blood cell of the frog and the crescent is so marked, especially when the latter is changing its shape, that it cannot be accidental. The true semilunar shape which the crescent assumes is certainly very curious and interesting; but it only takes this shape when the granules are collected quite in the centre, and the cell is consequently very markedly spindle-shaped as a mechanical result of the force of the blood current If you apply the methylene blue stain to a preparation of fresh blood first to one side of the cover-slip and then to the other, so as to create currents in opposite directions, you can make the crescent semilunar in either direction in which the ends are wafted by the current created by the stain.

4. The granules in Laveran's bodies occasionally undergo a " "swarming" movement, which Laveran compared to "the disturbance of boiling water." The same movement was discovered by us in the normal human leucocyte, and it can readily be produced artificially. The importance of this "swarming" movement cannot be overestimated. From the moment when we found swarming in the leucocyte it became clear that Laveran's bodies and the white cells are closely allied, and that one is no more likely to be a parasite than the other. "Swarming" is, confessedly, one of the most characteristic attributes of Laveran's bodies; it is a sign of life. The "swarming" movement, therefore, the boiling water movement of Laveran, on which the plasmodists rely most confidently for the identification of Laveran's bodies as parasites, if it is conclusive evidence of anything, is merely evidence that they are related to the white cell. The proof is clinched by the discovery of the same swarming movement in the normal protoplasmic cells and granules in the liver and spleen of frogs.

The same thing may be said with regard to the development of cilia or flagella in connection with Laveran's bodies. In view of the elementary truth that ciliary and flagellate varieties of pseudopods are known to be present and to constitute normal forces in the circulation in plants and animals, there does not appear to be any reason why they should be described as signs of parasite life only when they occur in connection with Laveran's bodies in the fever blood of malaria.

We now see that none of the qualities of Laveran's bodies warrant the assumption that they are parasites. On the contrary, they have their complete analogues in the cells of the blood both of man and of animals. Moreover, they are not present in the blood in a considerable proportion of cases of malarious fever of the most fatal and chronic types. They cannot therefore be the cause of the fever. If Laveran's bodies were the cause of the fever in malaria the plasmodists would surely have been able before now io isolate them in pure culture and to demonstrate their existence outside the human body. Dr. Patrick Manson states that "the intravenous injection of blood containing this parasite into a a healthy individual is followed by the multiplication of the parasite in the blood of the person inoculated, and also by the occurrence of the characteristic fever." There can be no justification for employing intravenous injections of malarial blood in the human subject, but during our investigation we did employ them repeatedly in dogs and in monkeys. When they produced any effect at all it was to give rise to a sharp attack of fever, which passed off on the same day, and was rot accompanied by the formation, much less by the multiplication, of Laveran's bodies in the blood. According to my experience, which is, however, confined to cholera cases, the intravenous injections of healthy blood or of saline fluid produce similar effects in man. They give rise to ague and fever, but the symptoms so produced by the saline fluid are most assuredly not malarial. From an examination of a very large number of spleens and livers of frogs, which are always healthy, we have come to the conclusion that frogs are immune against malaria. We therefore fed one of our fever patients for several days on the spleens and livers of frogs, and gave him daily subcutaneous injections of frog's serum. This treatment had no effect in arresting the attacks of fever, as it might have been expected to have had if the cause of the disease had been $(a)$ a parasite in the blood against which the frog is immune, or $(b)$ a defect in the spleen of the same nature as those defects of the thyroid gland which produce diseases which can be remedied by thyroid feeding.

Enough has been said to show that Laveran's bodies are not animal parasites living in the blood. The relationship between them and the normal blood cells is well brought out in this table, which makes it perfectly clear that in every phase of their existence Laveran's bodies have their analogue in normal blood. We have dealt with three forms of blood cells :

(a) Those of the flog, in which development is slow and easy to follow.

(b) Those of the healthy human subject, in which development is so rapid that it is difficult to trace except in the way of analogy with the frog.

(c) Those of the malarious human subject (Laveran's bodies), which are diseased or degenerate, but in which the development is not so rapid as in the healthy human blood cells, and in which the analogy to normal human blood cells on the one hand and the frog's blood cells on the other can be traced without difficulty.

A study of Laveran's bodies renders many points in the physiology of the blood clear and distinct. Thus, their intracorpuscular stage_indicates that the human white blood 


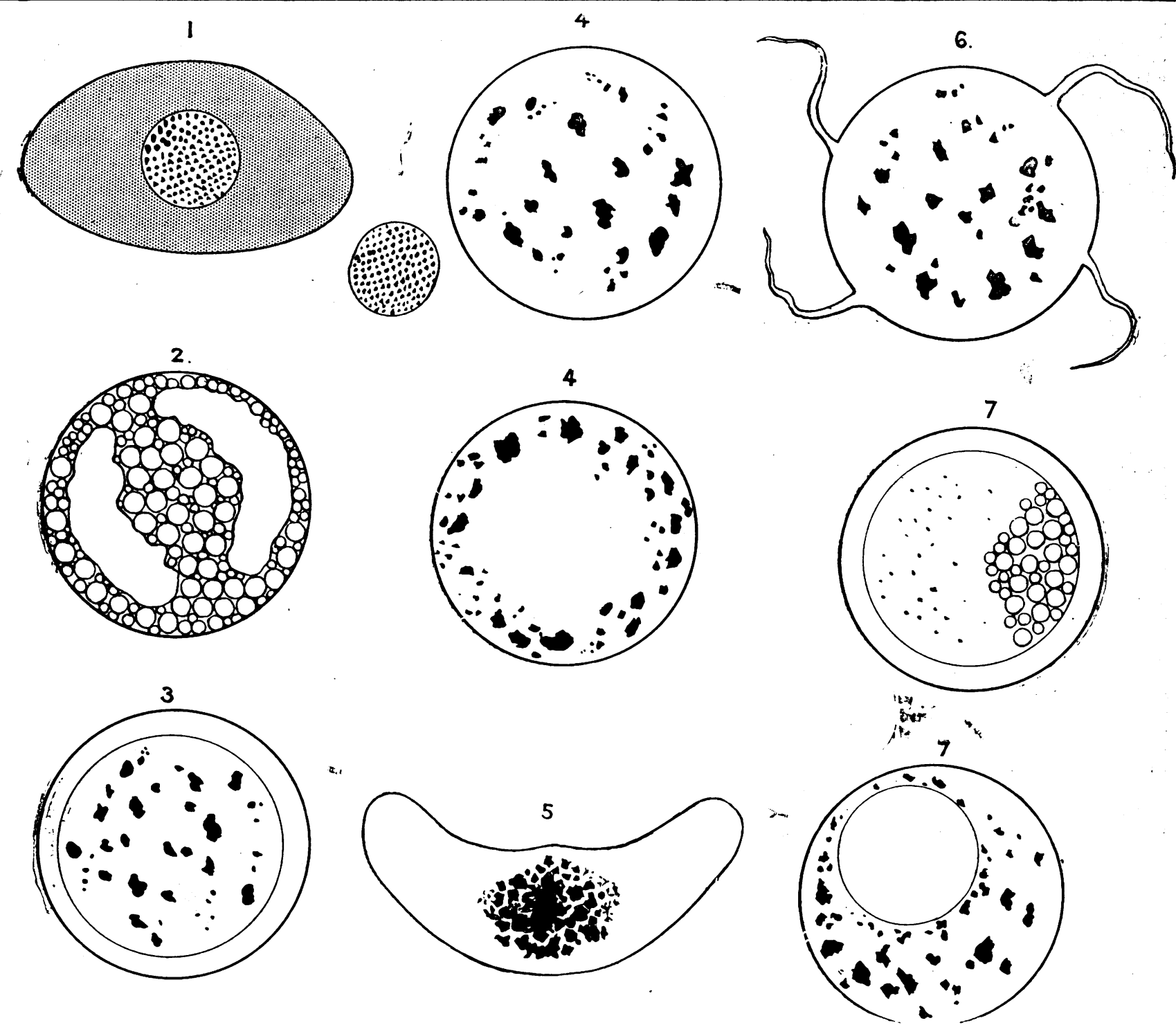

Wig. r.-Red and white blood cells of frog. From Landois and Stirling's Physiology. Fig. 2.- Leucocyte of human blood. Diagram. Fig.3.Intracorpuscular body of Laveran. Drawn from life. Fig. 4.-Extracorpuscular body of Laveran. The pigment-like gra nules are spread about. Drawn from life. Fig. 4 (2).-Intracorpuscular body of Laveran. The pigment-like bodies are spread out to the edge of the cell. Drawn from life. Fig. 5.- Extracorpuscular body of Laveran: the crescent. This cell is spindle-shaped, because the granules are collected diseased. Drawn from life. Fig. 7 (2).-Cell partially diseased. Drawn from life.

Explanatory Note on the Photographs of Blood Cells and of Laveran's Bodies.-The term "Laveran bodies" has been employed for convenience, without reference to the' school which claims to have discovered them. The striking points in the above groups of blood cells are: ( $a$ ) The re semblance between the white cell and the nucleus of the red cell of the frog's blood. (b) The resemblance between the red blood cell of the frog and the crescent. (c) "Swarming" was seen in Figs. 2, 4, 4 (2), 6, 7, and 7 (2). (d) In Figs. 7 and 7 (2) the cells are only partially diseased. In Fig. 7 the cell contained a number of healthy protoplasmic cellules, such as are seen in the healthy leucocyte along with the usual black pigment-like granules which are met with in Laveran's bodies. In Fig. 7 (2) the cell contains a clear nuclear space like the nuclear spaces in the leucocyte.

cell begins life as an intracorpuscular body. The extracorpuscular stage is synonymous with the free and independent existence of the leucocyte. The crescents indicate the spindle shape of the red cells, which is normal in the frog and other lower animals; and the swarming movement, and the development of ciliary or flagellate pseudopods affords strong presumptive evidence that these circulatory forces, which are recognised conditions of the circulation in animals and plants, are regularly at work in the capillary system of man.

It only remains to state or reaffirm the source of origin of Laveran's bodies and to consider the theories of malarious fever based upon it to the extent to which it is warranted by actual facts. The spleen is undoubtedly the organ mainly concerned in the production or elaboration of the white blood cells, though other blood glands may assist in the process. We have just seen that the analogy between Laveran's bodies and human blood cells as well as those of the frog, which are manufactured in the liver and spleen, is close and absolute, and, in the third place the spleen is invariably diseased in malarious poisoning. From these three facts it is evident that the Laveran's bodies are mostly the kind of degenerate blood cells which are turned out by the spleen when it is dragged down from its high pinnacle of evolution by malaria. The precise manner in which malaria acts upon the spleen can only be determined by future observation. All we know at present is that in certain localities and climates which we call malarious enlargement of the spleen with fever and 
anæmia is endemic, just as we know that in other localities and climates enlargement of the thyroid gland with bronchocele and various constitutional symptoms, and in others again stone in the bladder, are endemic.

Our theory of malarial fever, then, is that it is produced by the action of malaria on the spleen, and that the production of Laveran's bodies, which are not found in anything like all cases of malarial fever, is one of the effects of the distempered condition of the spleen-of splenitis. Several facts point irresistibly to this conclusion: for instance, congestion of the spleen characterised by enlargement or tenderness, or both, is the most constant of all the symptoms of rnalarious poisoning. Secondly, it is the sign on which reliance may most safely be placed in making the diagnosis of the malarial origin of fever, and if it is not present it is generally well to consider the advisability of excluding malaria as the cause. In the third place, the only drug which can be trusted to cure enlargement of the spleen from malaria and the fever which springs from it is quinine, and it restores equally those cases in which Laveran's bodies are and those in which they are not found in the blood. Lastly, when Laveran's bodies are present they attest in terms which are unmistakable that the spleen is the organ primarily affected by malaria.

The first effect of malaria on the spleen is to disorder its functions, and the patient gets fever, sometimes markedly periodic, sometimes not. It must not be forgotten that the temperature of the blood in health shows regular diurnal variations between $97^{\circ}$ and $99^{\circ}$, which depend to a large extent upon the periodic nocturnal inactivity and the diurnal activity of the spleen. The periodic attacks of fever are amply accounted for by the view that they are exaggerations of the diurnal activity of the spleen. The disturbance of its functions may cause the diurnal variations to be exaggerated daily-quotidian ague, or every alternate day-tertian ague, or every third day-quartan ague. As the disorder of the spleen increases its functions of regulating the diurnal variations of temperature become less clearly pronounced, they are as it were lost in the disease, and as a consequence the attacks of fever seldom maintain periodic regularity for any length of time, and they become irregular. If the irritation of the spleen is very great, or if it is accompanied by derangement of important organs like the brain, the liver, the stomach, or the bowels, the fever may be irregular or continuous from the onset.

As the disease progresses the spleen is no longer able to elaborate its full complement of perfect white cells. It manufactures a certain number of imperfectly developed or abnormal cells, which pass into the blood, and these are Laveran's bodies. The symptoms produced by the presence of these bodies in the blood are easy to understand; they are symptoms of disordered nutrition. Instead of receiving their normal supply of life-giving white cells all the tissues of the body get a proportion of Laveran's barren innutritious and not infrequently lifeless organisms. It is not surprising that a patient who has malarial fever is generally pale, and has headache or perhaps severe cerebral symptoms, with indigestion, constipation, and derangement of the liver, or that he feels very ill and becomes thin; the nutrition of every tissue and organ in the body suffers. Finally, when the enlargement of the spleen becomes, as we say, chronic, its functions as a blood-forming organ are entirely in abeyance; even the degenerate cells of Laveran are no longer produced and the anæmia becomes permanent and profound.

\section{ON THE VARIOUS EXPLANATIONS OF SPON- TANEOUS FRACTURE OF STONES IN THE BLADDER.}

By DEBOUT D'ESTRÉES, M.D., Contrexéville.

I Do not here wish to quote the old authors who have treated the interesting question of the spontaneous fragmentation of calculi in the bladder, nor to relate the facts which $I$ have observed since the first example came under my notice at Contrexéville in 1869 , which with many others I related at the International Congress of Medicine held at Brussels in 1875 .
I wish now to speak of what I have learnt on this question in my experience at Contrexéville, where I have practised since 1868. The explanations which have been offered by different authors of the mechanism of this spontaneous fragmentation have, in my opinion, the fault of being too exclusive, and they have been successively rejected. There are at least three ways of explaining the spontaneous fragmentation of calculi.

The first is the theory of my late master, Civiale-energetic contraction of a bladder the muscular tunic of which is hypertrophied.

Secondly, the exfoliation or rupture of the cracking of the peripheral layers of a large calculus, or of numerous small calculi which are no more moistened by urine either on account of their size or their number.

Thirdly, the bursting of the calculus caused by the expansion of its nucleus, according to the theory more particularly adopted by Dr. Ord.

The opinion of Dr. Civiale is not shared by many, and his theory would not apply to the great majority of the cases I have observed at Contrexéville, nor, amongst others, to those which Dr. Wilberforce Smith and Mr. Hurry Fenwick presented in 1890 to the Pathological Society. But this is not a reason for rejecting it altogether, and I give here an example in its favour.

A man, aged 70 , had been passing uric acid and small calculi for thirty years. He had been suffering for about a year from severe attacks of pain which had their seat in the region of the bladder, and which were always followed by the expulsion of fragments of calculi. He came to Contrexéville five years ago, and took from 3 to 4 pints of water daily without medical advice. He passed the voluminous fragments represented in Fig. $\mathrm{I}$.

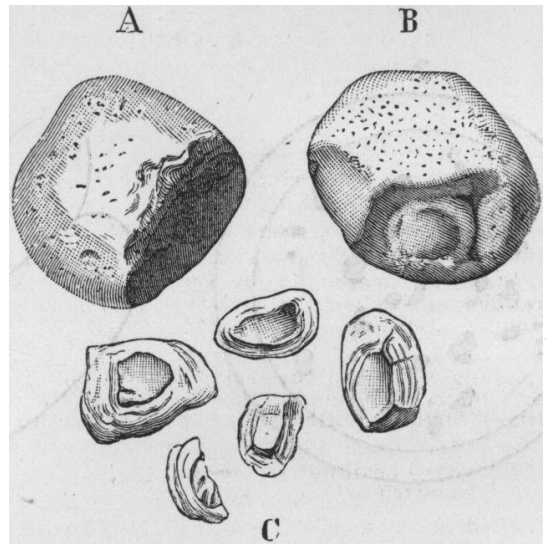

Fig. r.-Fragments expelled at Contrexéville, and two of the five calculi, one A integral the other B partially broken, removed by a suprapubic cystotomy.

The last of these fragments caused a violent attack of urethral fever, and the patient sent for me. The diagnosis was easy-vesical calculi not capable of passing by the natural channels. I ordered cessation of the hydromineral treatment, which I would never have advised had I been consulted from the first. An exploration showed the existence of numerous large calculi, which were shortly after removed by a suprapubic cystotomy by Dr. Segond at the Charite Hospital in Paris. We do not operate on patients at Contrexéville as a rule. The surgeon withdrew five calculi, two of which are represented in the above figure.

Three of these calculi were intact, but two presented the appearance of more or less recent fracture, and, as will be seen by Fig. 1, B, this fracture only appeared on one angle of the calculus. There could not be any question of exfoliation or bursting in this case, and it therefore seems to me to be but just to admit, with Civiale, the explanation of the phenomenon by the contraction of a hypertrophied bladder coinciding with the painful attacks experienced by the patient.

The theory of exfoliation applies more particularly to large calculi, of which the parts not moistened with urine dry up and retract, thereby producing cracks, which bring about the 